\title{
Point-of-Care Ultrasound for Identification of Ruptured Infrarenal Abdominal Aortic Aneurysm
}

\author{
Anne Kelly Rhudy ${ }^{1}$, Shivik Patel $^{1}$, Alex Houser ${ }^{1}$, and Malachi Sheahan ${ }^{1}$ \\ ${ }^{1}$ LSU Health New Orleans
}

January 1, 2022

\begin{abstract}
Ruptured abdominal aortic aneurysms are associated with high mortality rates and require prompt diagnosis with subsequent intervention. CT scan is considered the gold-standard for diagnosis, however, in the acute setting ultrasound may be a reasonable diagnostic test for certain patients. We report a case that demonstrates the utility of bedside ultrasound of rAAA for a patient in extremis. Also, we provide a brief review of literature for the diagnosis of rAAA with ultrasound.
\end{abstract}

\section{Title}

Point-of-Care Ultrasound for Identification of Ruptured Infrarenal Abdominal Aortic Aneurysm

\section{Authors}

Anne Kelly Rhudy MD MPH,

Division of Vascular Surgery, Louisiana State University Health Sciences, New Orleans, LA

Shivik Patel MD

Division of Vascular Surgery, Louisiana State University Health Sciences, New Orleans, LA

Alex Houser BS

Division of Vascular Surgery, Louisiana State University Health Sciences, New Orleans, LA

Malachi Sheahan MD

Division of Vascular Surgery, Louisiana State University Health Sciences, New Orleans, LA

\section{Correspondence}

Anne Kelly Rhudy

Department of Surgery

LSUHSC School of Medicine

1542 Tulane Ave, 7th Floor

New Orleans, LA 70112

Email: arhudy@lsuhsc.edu

\section{Abstract}


Ruptured abdominal aortic aneurysms are associated with high mortality rates and require prompt diagnosis with subsequent intervention. CT scan is considered the gold-standard for diagnosis, however, in the acute setting ultrasound may be a reasonable diagnostic test for certain patients. We report a case that demonstrates the utility of bedside ultrasound of rAAA for a patient in extremis. Also, we provide a brief review of literature for the diagnosis of rAAA with ultrasound.

\section{Keywords}

ruptured infrarenal abdominal aortic aneurysm, ultrasound, point of care

\section{Background}

Despite major advances in surgical techniques, ruptured abdominal aortic aneurysms a still carry high mortality. The overall mortality of ruptured abdominal aortic aneurysm (rAAA) is approximately $70 \%$ including in and out of hospital deaths with approximately half dying prior to hospital presentation. ${ }^{1}$ Surgical mortality remains at least $40 \%$ for those that do make it to the operating room. A missed diagnosis of rAAA is lethal. Established poor preoperative mortality risk factors include old age, hypotension, and elevated creatinine at presentation. ${ }^{1}$ Our case study focuses on a patient with known infrarenal abdominal aortic aneurysm (AAA) who was brought into the emergency department after cardiac arrest. A point-of-care (POC) ultrasound was obtained which confirmed rAAA. Bedside ultrasound can be a useful diagnostic tool, especially in patients in extremis or in those who are unable to provide a history. Obtaining a CT scan can be difficult in hemodynamically unstable patients and thus a POC ultrasound may be an expedient, non-invasive tool to guide clinical decision making in the acute care setting.

\section{Case Report}

Patient MC was a 78-year-old female with a past medical history of diabetes, obstructive lung disease, hypertension, cirrhosis with esophageal varices and known AAA who presented to the emergency department after cardiac arrest from her nursing home. She was found unresponsive, hypoglycemic and with pulseless electrical activity. Dextrose was given and ACLS initiated with return of spontaneous circulation after 18 minutes. She required vasopressor support. Ultrasound findings in the emergency department were concerning for rAAA and vascular surgery was consulted. After discussion with family, in the setting of the patient's cardiac arrest in the field with an unknown anoxic time we pursued palliative options.

\section{Ultrasound Images}

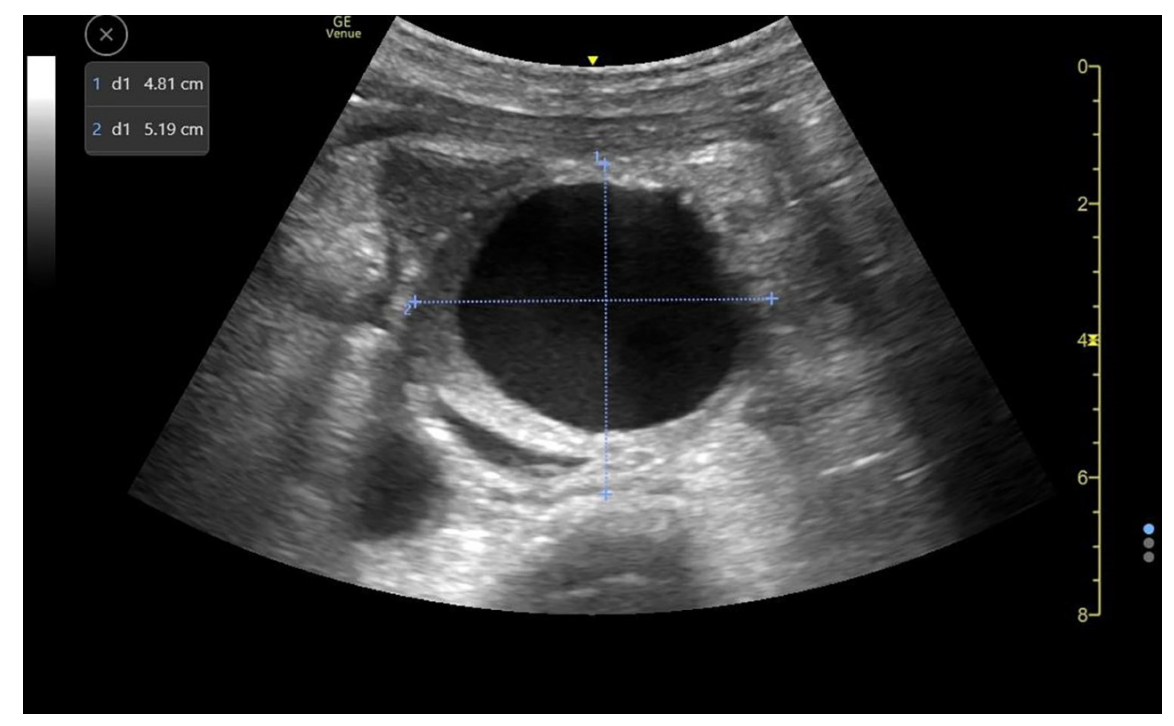

Figure 1: Transverse view demonstrating $5.19 \mathrm{~cm}$ AAA at the level of the lowest left renal 
artery

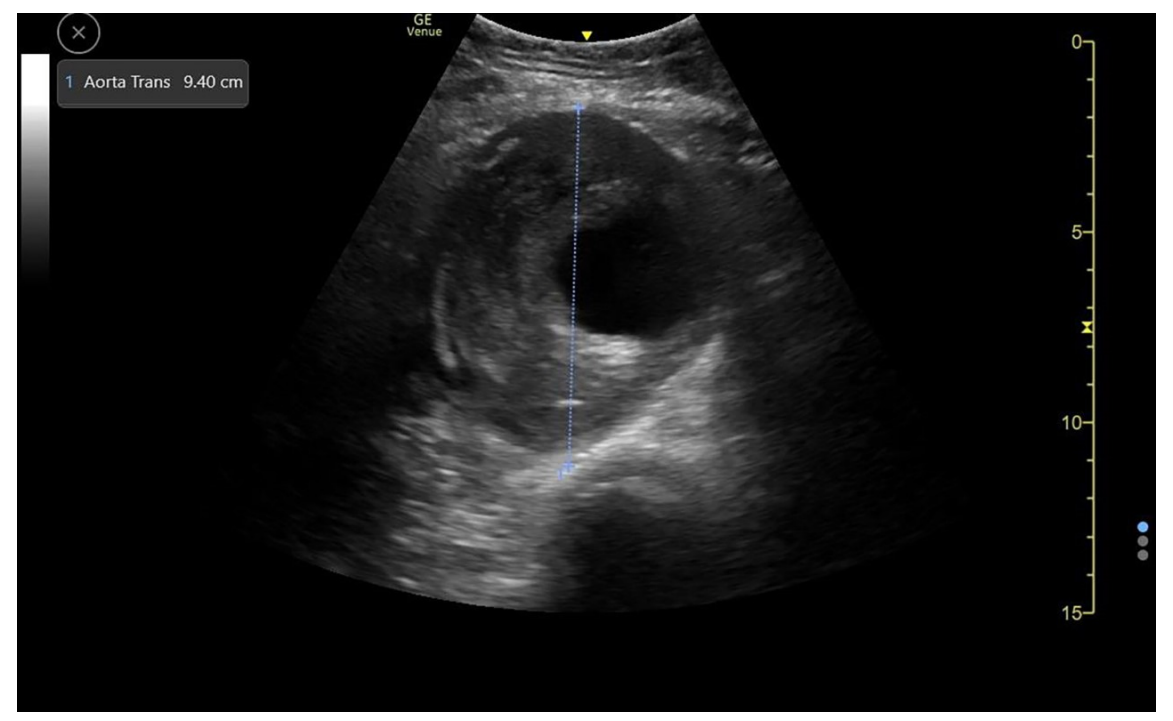

Figure 2: Transverse view showing $4.4 \mathrm{~cm}$ AAA with periaortic stranding along the posterolateral wall consistent with $\mathrm{rAAA}$

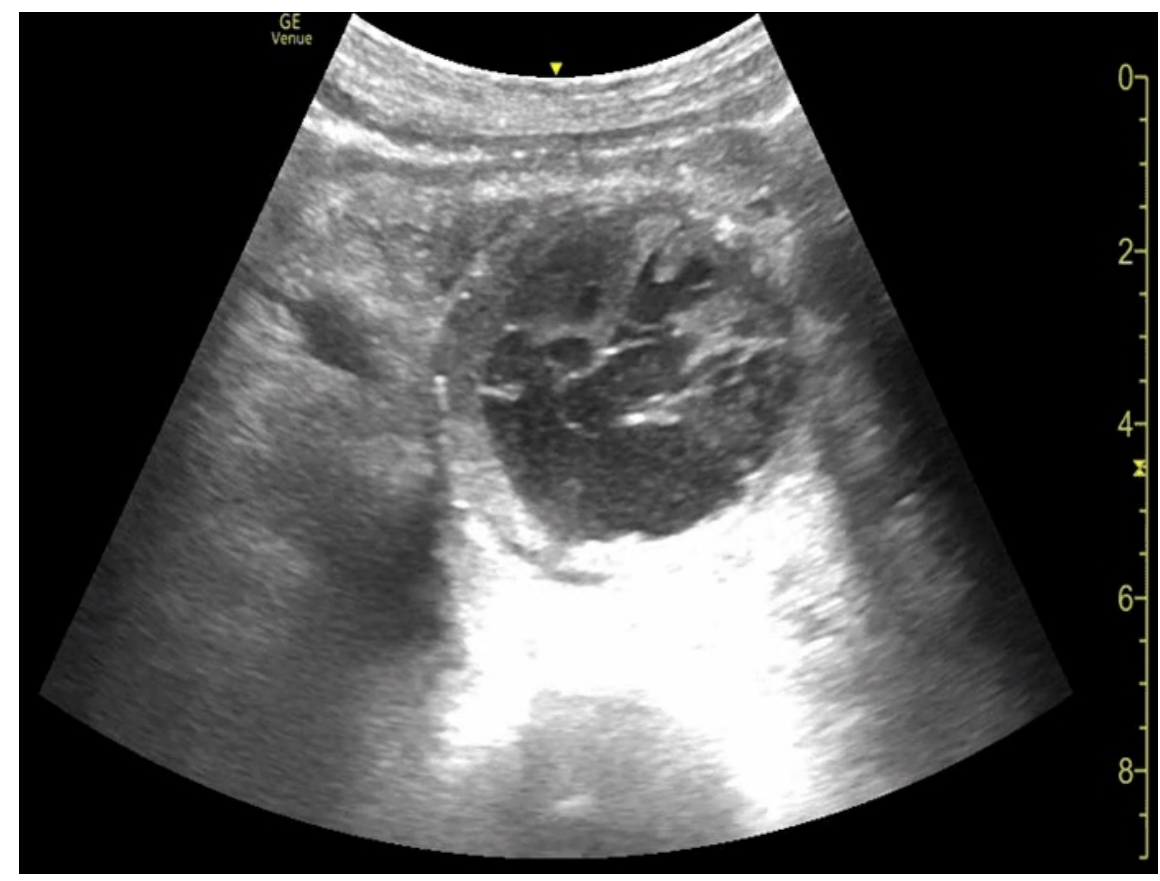

Figure 3: Transverse view with heterogenous intramural thrombus, with disruption of retroperitoneal planes and hematoma consistent with rAAA 


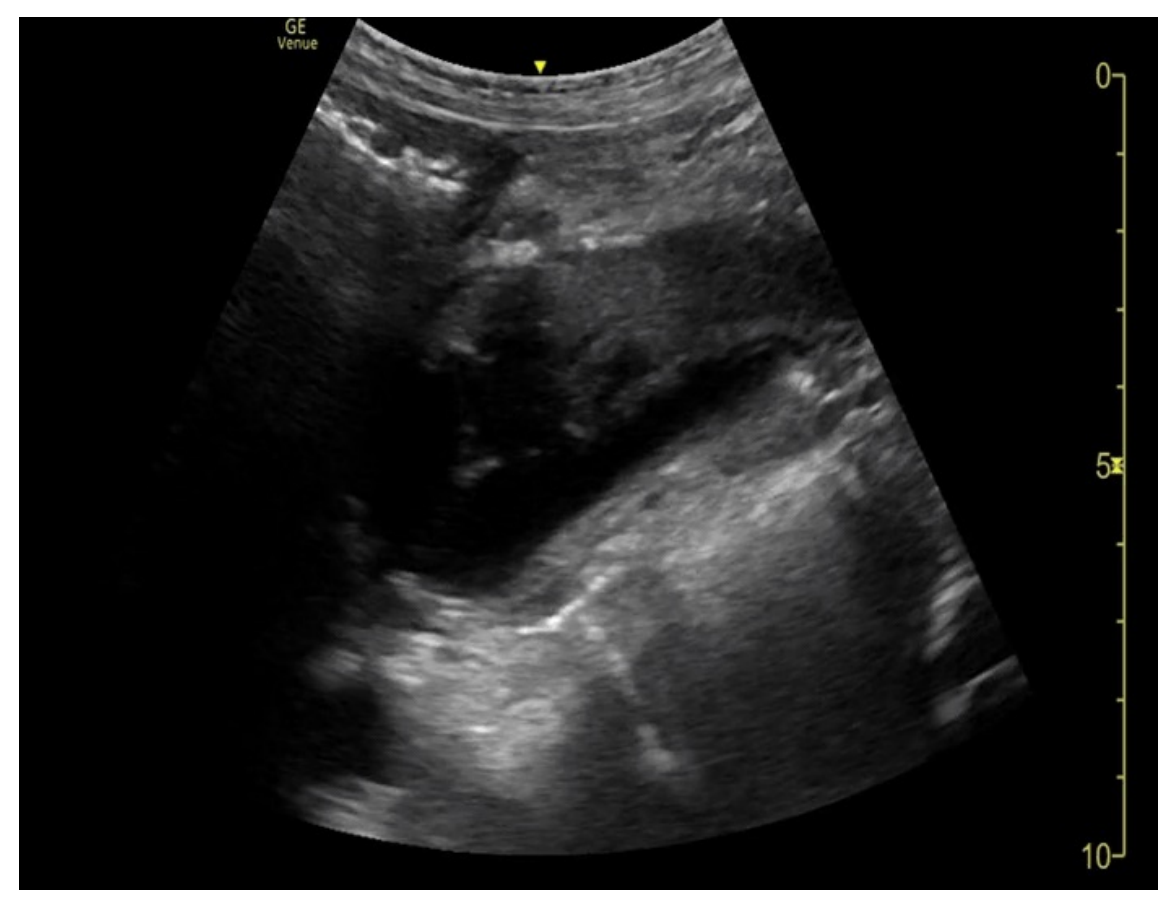

Figure 4: Sagittal view of floating intramural hematoma with dissection flap. Disrupted retroperitoneal tissue planes inferiorly

\section{Discussion}

Without rapid intervention, rAAA carries a high mortality. Using ultrasound assessment of patients in extremis may decrease time to the operating room. Unfortunately, with our patient's co-morbidities and cardiac arrest in the field she was not a candidate for surgical repair. Nevertheless, it is important to appreciate the value of ultrasound findings of rAAA. Catalano and Siani discussed the sonographic findings of ruptured AAA in 2005. Their series included 29 patients with rAAA (either confirmed via surgery or CT scan). The findings consistent with rAAA are (1) AAA deformation, (2) Luminal thrombus inhomogeneity, (3) Clear interruption of the lumen thrombus, (4) Intraluminal floating thrombus layer, (5) Aneurysm wall interruption, (6) Para-aortic hypoechoic area adjacent to bleeding site, (7) Retroperitoneal hematoma, and (8) Hemoperitoneum. ${ }^{2}$ All of these signs, excluding AAA deformation, were noted in rAAA. There is discussion as to whether with improved sonographic technology, in the hands of an experienced user, if ultrasound could replace the need for pre-operative CT angiograms, especially in hemodynamically unstable patients.

Perioperative hypotension is a negative survival predictor for rAAA; therefore, delays in diagnosis, can increase time to operative repair and prolong hypotensive time. In 2014, Reed and Cheung studied the effect of an emergency ultrasound program to expedite time to diagnosis. Although their study may have been underpowered for statistical significance, there was an overall decrease in time to diagnosis (56 minutes vs 111 minutes). ${ }^{3}$ This is an area of further inquiry in the current literature. Excluding technical considerations, a drawback of ultrasound can be the difficulty in assessing relevant visceral and renal artery anatomy for endovascular. Although a significant portion of open AAA repairs will require supraceliac clamping, it can be more difficult to assess distal targets such as iliac artery involvement. ${ }^{4}$ With ultrasound being increasingly employed for diagnosing of complex and urgent pathology, future studies are needed to address its use for pre-operative planning in the acute setting.

Within the current literature, more comparative studies need to be performed before ultrasound can replace CT angiogram as diagnostic gold standard for rAAA. Nevertheless, POC ultrasound facilitates swift clinical 
decision making for immediate patient care, with the added benefit of lower cost.

\section{References}

[1] Hoornweg LL, Storm-Versloot MN, Ubbink DT, Koelemay MJW, Legemate DA, Balm R. Meta-analysis on mortality of ruptured abdominal aortic aneurysms. European journal of vascular and endovascular surgery: the official journal of the European Society for Vascular Surgery. 2008;35(5):558-570.

[2] Catalano O, Siani A. Ruptured abdominal aortic aneurysm: categorization of sonographic findings and report of 3 new signs. Journal of ultrasound in medicine: official journal of the American Institute of Ultrasound in Medicine. 2005;24(8):1077-1083.

[3] Reed MJ, Cheung L-T. Emergency department led emergency ultrasound may improve the time to diagnosis in patients presenting with a ruptured abdominal aortic aneurysm. European journal of emergency medicine: official journal of the European Society for Emergency Medicine. 2014;21(4):272-275.

[4] El-Sabrout RA, Reul GJ. Suprarenal or supraceliac aortic clamping during repair of infrarenal abdominal aortic aneurysms. Tex Heart Inst J. 2001;28(4):254-264.

\section{Figure Legends}

Figure 1: Transverse view demonstrating $5.19 \mathrm{~cm}$ AAA at the level of the lowest left renal artery

Figure 2: Transverse view showing $4.4 \mathrm{~cm}$ AAA with periaortic stranding along the postero-lateral wall consistent with rAAA

Figure 3: Transverse view with heterogenous intramural thrombus, with disruption of retroperitoneal planes and hematoma consistent with rAAA

Figure 4: Sagittal view of floating intramural hematoma with dissection flap. Disrupted retroperitoneal tissue planes inferiorly

\section{Data Availability Statement}

The data that support the findings of this study are available on request from the corresponding author. The data are not publicly available due to containing information that could compromise the privacy of patients. 